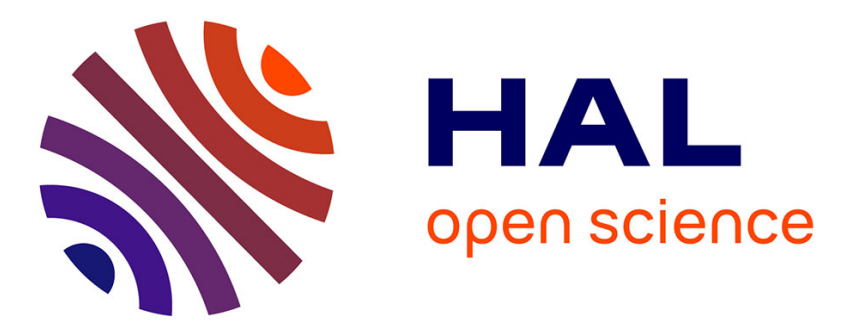

\title{
From PD to Nonlinear Adaptive Depth-Control of a Tethered Autonomous Underwater Vehicle
}

Divine Maalouf, Ivan Tamanaja, Eduardo Campos, Ahmed Chemori, Vincent Creuze, Jorje Torres, Lozano Rogelio

\section{- To cite this version:}

Divine Maalouf, Ivan Tamanaja, Eduardo Campos, Ahmed Chemori, Vincent Creuze, et al.. From PD to Nonlinear Adaptive Depth-Control of a Tethered Autonomous Underwater Vehicle. IFAC Joint conference, SSSC: Symposium on System Structure and Control, Feb 2013, Grenoble, France. lirmm-00778061

\section{HAL Id: lirmm-00778061 https://hal-lirmm.ccsd.cnrs.fr/lirmm-00778061}

Submitted on 6 Feb 2013

HAL is a multi-disciplinary open access archive for the deposit and dissemination of scientific research documents, whether they are published or not. The documents may come from teaching and research institutions in France or abroad, or from public or private research centers.
L'archive ouverte pluridisciplinaire HAL, est destinée au dépôt et à la diffusion de documents scientifiques de niveau recherche, publiés ou non, émanant des établissements d'enseignement et de recherche français ou étrangers, des laboratoires publics ou privés. 


\title{
From PD to Nonlinear Adaptive Depth-Control of a Tethered Autonomous Underwater Vehicle
}

\author{
D.Maalouf ${ }^{*}$ I.Tamanaja ${ }^{* *}$ E.Campos ${ }^{* *}$ A.Chemori ${ }^{*}$ V.Creuze $^{*}$ \\ J.Torres $^{* *}$ R.Lozano ${ }^{* *}$ \\ * LIRMM,Univ. Montpellier 2-CNRS, 161 rue Ada, 34392 Montpellier, France \\ (e-mail: ahmed.chemori@lirmm.fr) \\ ** Laboratoire Franco-Mexicain d'Informatique et Automatique, LAFMIA \\ UMI 3175 CNRS-CINVESTAV, Mexico (e-mail: Rogelio.Lozano@hds.utc.fr)
}

\begin{abstract}
This paper deals with an experimental comparison between a proportional derivative (PD) controller and an adaptive nonlinear state feedback one, both applied on a tethered autonomous underwater vehicle. The aim is to show the behavior of the closed loop system in the nominal case for each of these two controllers, and then to test their robustness towards some parameters changes. The PD on one hand has a good performance for systems with an unknown model. The adaptive control law on the other hand is known to adjust the unknown parameters of the plant in order to converge to the desired trajectory. This study shows experimental results performed using each of the above mentioned control laws.
\end{abstract}

Keywords: Underwater robotics, Depth control, Parameters uncertainty, PD controller, Adaptive control.

\section{INTRODUCTION}

Underwater Vehicles have gained an increase interest in the last decades given the multitude of operations they can perform in various fields. Different control techniques have then been proposed and applied in order to deal with the various challenges arising from the nonlinearities and time varying behavior of the vehicle's dynamics. An overview of the main control techniques for underwater vehicles can be found in Fossen [2002], Yildiz et al. [2009] and Yuh [2000]. Control design methods can be either linear or nonlinear. In the linear techniques PID controllers are still very common but they do not guarantee system's position stability given the highly nonlinear behavior of the underwater vehicle (Yildiz et al. [2009]). Some more advanced linear techniques such as adding an acceleration feedback to the PID in order to make the vehicle less sensitive to external disturbances or using least square regulators to track time varying reference trajectories can be found in Fossen [2002]. These techniques can also be suitable for uncertain linear systems disturbed by additive white noise and the presence of some immeasurable states. However, limitations when using such techniques are still present and that is why more research has been oriented recently towards nonlinear control schemes. In this latter category, various methods were successfully applied to underwater vehicles such as sliding mode control: Fossen and Foss [1991] and Healey et al. [1995] and nonlinear adaptive control: Antonelli [2006], Antonelli et al. [2001] and Fossen and Sagatun [1991]. The former is a robust control scheme towards parameters uncertainties and external disturbances. Its main drawback is the chattering phenomenom which directly affects the thrusters leading to a high energetic consumption and possible damages. The latter method, which is model based, ensures the stability of the system through a suitable parameters estimation (i.e. not necessarily the "true" values of the parameters) (Slotine and Weiping [1991] ). Exper- imental results showing the efficiency of this method have been performed on the underwater vehicle ODIN in Antonelli et al. [1999]. In Maalouf et al. [2012] the first implementation of an $\mathscr{L}_{1}$ adaptive controller on an underwater vehicle was presented with experimental results. Other nonlinear methods based on intelligent control have also been proposed. They include for instance neural networks and fuzzy logic controllers. Neural networks is a powerful tool since it has a parallel structure, applicability to hardware implementation and multivariable nature. However, training time is too long especially in the presence of parameters changes which makes it hard to be implemented in real-time applications (Shaw [1998]). Fuzzy logic controllers have been tested in simulations such as in Chang et al. [2003], but they require many trial and error cycles to achieve the desired performance (Kim and Yuh [2001] ). To combine the advantages of the previous two mentioned controllers, a study supported by simulations has been provided in Kim and Yuh [2001], where a neuro-fuzzy controller is proposed but no experimental results have been carried out yet to validate the theory.

We can find in literature some comparisons among control laws through simulations, Antonelli [2007] and Campa et al. [1998] but few studies perform these comparisons empirically. In Smallwood and Whitcomb [2002], adaptive and fixed-model based controllers have been compared with a PD controller in terms of tracking performance in the presence of thruster saturation and modeling errors. The presented experimental results show that model based controllers exhibit a bad performance in presence of modeling errors compared to the classical Proportional Derivative (PD) controller, while adaptive ones compensate properly these uncertainties. Thrusters saturation deteriorates the performance of all the controllers.

In this paper we will experimentally compare a PD controller with an adaptive nonlinear state feedback one. We present experimental results for one degree of freedom, namely the depth. 
Our contribution lies in the presented experimental comparative study that shows the differences in the robot's behavior regarding each applied method in terms of settling time, and trajectory following tested in various conditions. On one hand, the PD controller was chosen because it is considered the basic one used among the non adaptive schemes and on the other hand, the nonlinear adaptive state feedback controller is the standard adaptive one. Comparing these two methods would give us an idea about the benefits of using an adaptive scheme. Two tests of robustness were carried out. The first one deals with the robustness towards parameters changes. It was technically performed by changing the buoyancy of the vehicle by adding floating balls on the top of the submarine as shown in Fig. 6 . The second one deals with the robustness towards punctual external disturbances. The experiments have been conducted using the tethered autonomous vehicle Triton-PR entirely designed and built by the LAFMIA (Franco-Mexican Laboratory specialized in Informatics and Automatic Control). This study has been performed to highlight the importance of applying more advanced control techniques in underwater robotics applications and it can be easily generalized to more complex situations.

The paper is organized as follows: in the second part of the paper we present the features of the vehicle we have used, and we remind the basic principles used in its modeling. In the second part, we present theoretical aspects of both controllers that we aim to compare. In the last part of the paper, we detail the real-time experiments that we have conducted, we compare the obtained results, and finally we conclude.

\section{TRITON-PR PROTOTYPE: DESCRIPTION AND DYNAMICS}

\subsection{Description of the experimental platform}

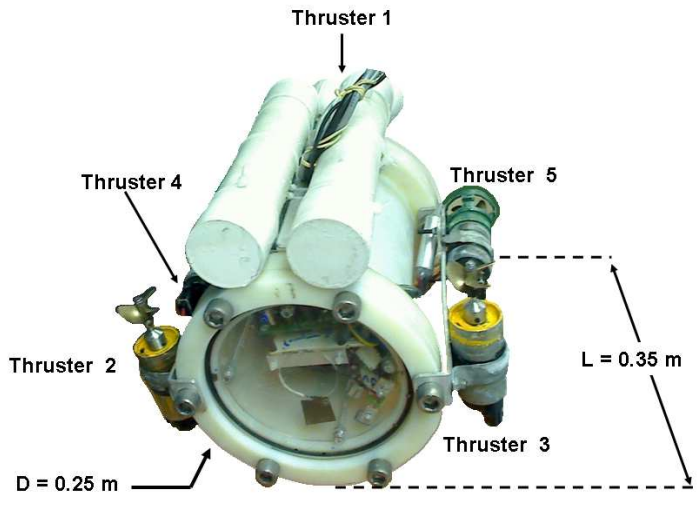

Fig. 1. View of the Triton-PR submarine and its five thrusters.

The Triton-PR submarine (cf. Fig.1) is an underactuated vehicle, whose propulsion system consists of five thrusters (the thrusters were built using DC motors with metal propellers). These actuators allow controlling the vehicle's orientation (yaw, pitch, roll) as well as its cartesian position ( $\mathrm{x}$ and $\mathrm{z}$ ). The yaw control is provided thanks to differential speed control of the thrusters 4 and 5 (cf. Fig.1). Pitch control is obtained similarly using thrusters 1, 2 and 3, whereas the roll control employs thrusters 2 and 3. The translational motion along the $\mathrm{z}$ axis is regulated by decreasing or increasing the combined speed of thrusters 1, 2 and 3. Similarly, translational motion along the $x$ axis is obtained thanks to thrusters 4 and 5 . The different forces induced by the five thrusters are illustrated in Fig. 2.

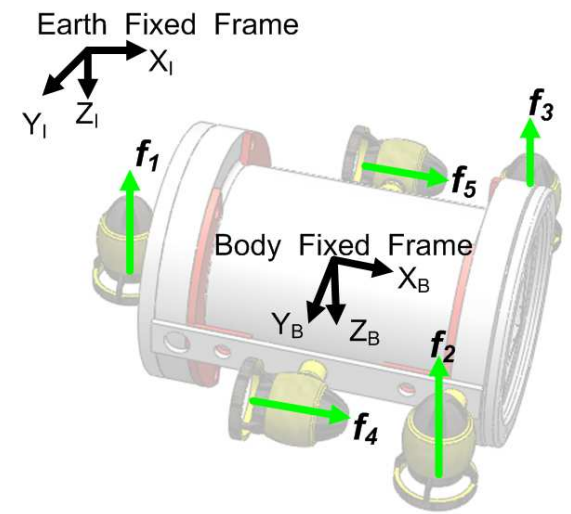

Fig. 2. Position and orientation of the forces induced by the five thrusters.

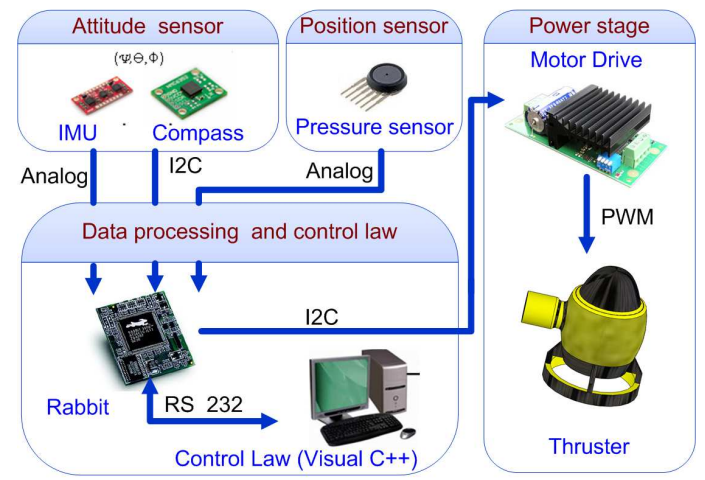

Fig. 3. Hardware architecture of Triton-PR prototype.

The prototype is equipped with various sensors for position and orientation measurements. An analog 6 DOF IMU (Inertial Measurement Unit) is used for roll and pitch, a compass module (HMC6352, with 0.5 degree heading resolution, and an I2C Interface) is used for the attitude, and a silicon pressure sensor (MPX5999D on-chip signal conditioned, 0 to $150 \mathrm{psi}$ ) for depth measurement. To process the sensors' data and insure communication between the control PC, the sensors and the power stage, a RabbitCore RCM3400 board is used. This latter includes a Rabbit 3000 microcontroller operating at $29.4 \mathrm{MHz}$. Once data are processed by the PC, the computed values are sent to the rabbit to activate the motors' power (controlled by Pulse Width Modulation thanks to MD03 -24V 20A H Bridge Motor Drives with I2C Interface). Real time communication is provided through a RS232 link. Fig. 3 shows a schematic view summarizing the various components of the vehicle's hardware and their interactions.

\subsection{Dynamic modeling of the system}

Throughout the paper, the variables in bold represent matrices and the others represent scalars. 
By considering the inertial generalized forces, the hydrodynamic effects, the gravity, and buoyancy contributions as well as the effects of the actuators (thrusters), the dynamic model of an underwater vehicle in matrix form, using the SNAME notation and the representation proposed in Fossen [2002], is written as:

$$
\begin{gathered}
\dot{\eta}=J(\eta) v \\
M \dot{v}+C(v) v+D(v) v+g(\eta)=\tau
\end{gathered}
$$

where $\boldsymbol{v}=[u, v, w, p, q, r]^{T}, \boldsymbol{\eta}=[x, y, z, \varphi, \vartheta, \psi]^{T}$ are vectors of velocities (in the body-fixed frame) and position/Euler angles (in the earth-fixed frame) respectively. $\boldsymbol{J}(\boldsymbol{\eta}) \in \mathbb{R}^{6 \times 6}$ is the transformation transformation matrix mapping from the bodyfixed frame to the earth-fixed one. The model matrices $\boldsymbol{M}, \boldsymbol{C}$ and $\boldsymbol{D}$ denote inertia (including added mass), Coriolis-centripetal (including added mass), and damping respectively, while $\boldsymbol{g}$ is a vector of gravitational/buoyancy forces. $\tau$ is the vector of forces and torques. The experiments have been performed in a small pool and hence, external disturbances such as currents were not taken into account in the model (1). In the case of our study, the vehicle used will be moving at low velocities, which makes the Coriolis terms negligible. Therefore the dynamics (1) can be rewritten as:

$$
M \dot{v}+n(v, \eta)=\tau
$$

with $n(v, \eta)=D(v) v+g(\eta)$

Our vehicle is equipped with 5 thrusters controlling 5 degrees of freedom as described above. The control input dimension will therefore be limited to 5 and is given by:

$$
\tau=T K u
$$

where $\boldsymbol{u} \in \mathbb{R}^{5 \times 1}$ is the vector of control inputs, $\boldsymbol{K} \in \mathbb{R}^{5 \times 5}$ is the force coefficient diagonal matrix, and $\boldsymbol{T} \in \mathbb{R}^{6 \times 5}$ is the actuators configuration matrix. An identification test has been performed to determine the relationship between the input voltage to the thrusters and the generated force.

\section{PROPOSED CONTROL SCHEMES}

In this section the proposed two controllers are presented: the proportional derivative and the adaptive nonlinear state feedback. They are implemented on the prototype described in section 2 in order to perform trajectory following along the $\mathrm{z}$ axis. The full model of the system has been previously presented but in this study the controller will be applied to the dynamics of the vehicle in depth.

\subsection{Proportional Derivative (PD) controller}

This control strategy is based on separated saturation functions. The objective of this control law, based on the use of saturation functions, is to limit aggressive control signals sent to the thrusters that may damage them. Therefore, the control input in depth is given by:

$$
\tau_{z}=-\sigma_{b_{z_{2}}}\left(k_{z_{2}} \dot{z}\right)-\sigma_{b_{z_{1}}}\left(k_{z_{1}}\left(z-z_{d}\right)\right)
$$

where $k_{z_{1}}, k_{z_{2}}$ are positive constants gains, representing respectively the proportional and the derivative gains. $\sigma_{b_{z_{1}}}$ and $\sigma_{b_{z_{2}}}$ are saturation functions. It has been proven in Teel [1992] that this control input can ensure a global stabilization of the system and that there exists a time large enough such that $\left(z-z_{\text {des }}\right) \rightarrow 0, \dot{z} \rightarrow 0$, as $t \rightarrow \infty$. The gains of the PD controller were empirically tuned to minimize the ISTSE criterion (Integral of Time Multiplied by squared Error) while compromising between the stability and a fast convergence.

\subsection{Adaptive Nonlinear State Feedback Controller}

The adaptive state feedback controller is a state feedback controller with an adaptation part. It provides an online estimation of the unknown model parameters in order to ensure a proper trajectory following Fossen [2002]. The control law is given by:

$$
\boldsymbol{\tau}=\hat{\boldsymbol{M}} \boldsymbol{a}^{\boldsymbol{b}}+\hat{\boldsymbol{n}}(\boldsymbol{v}, \boldsymbol{\eta})
$$

where the hat symbol denotes the parameter estimates, $\boldsymbol{a}^{\boldsymbol{b}}$ is the body frame commanded acceleration and $\hat{n}(\boldsymbol{v}, \boldsymbol{\eta})$ is the estimate of $\boldsymbol{n}(\boldsymbol{v}, \boldsymbol{\eta})$ in (2). Since the dynamic model is linear in its parameters, the adaptive control law is then written as:

$$
\tau=\Phi\left(a^{b}, v, \eta\right) \hat{\theta}
$$

where $\boldsymbol{\Phi}$ is the regressor matrix and $\hat{\boldsymbol{\theta}}$ is the vector of the estimated parameters. To guarantee that error converges to zero, the commanded acceleration in the inertial frame $\boldsymbol{a}^{\boldsymbol{n}}$ is chosen as the following proportional derivative (PD) control:

$$
a^{n}=\ddot{\eta}_{d}-K_{d} \dot{\tilde{\eta}}-K_{p} \tilde{\eta}
$$

with $\tilde{\boldsymbol{\eta}}=\boldsymbol{\eta}-\boldsymbol{\eta}_{\boldsymbol{d}}, \dot{\tilde{\boldsymbol{\eta}}}$ the first derivative of $\tilde{\boldsymbol{\eta}}, \boldsymbol{\eta}_{\boldsymbol{d}}$ is the desired trajectory and $\ddot{\boldsymbol{\eta}} \boldsymbol{d}_{\boldsymbol{d}}$ the desired acceleration

Combining equations (1) and (7), the expression of $\boldsymbol{a}^{\boldsymbol{b}}$, the acceleration in the body-fixed frame becomes:

$$
\boldsymbol{a}^{\boldsymbol{b}}=\boldsymbol{J}^{-\mathbf{1}}\left(\boldsymbol{a}^{\boldsymbol{n}}-\boldsymbol{J} \boldsymbol{v}\right)
$$

The parameter update law is given by:

$$
\dot{\hat{\theta}}=-\Gamma \Phi^{T}\left(a^{b}, v, \eta\right) J^{-1} y
$$

where $\boldsymbol{\Gamma}$ is the adaptation gain diagonal matrix, $\boldsymbol{J}$ the transformation matrix and $\boldsymbol{y}$ the combined error defined as:

$$
\boldsymbol{y}=c_{0} \tilde{\boldsymbol{\eta}}+c_{1} \dot{\tilde{\boldsymbol{\eta}}}
$$

with $c_{0}$ and $c_{1}$ being constant gains chosen to ensure the stability of $\boldsymbol{y}$. In Fossen [2002] the complete proof of stability stating that the convergence of the position error to zero is guaranteed by applying Barbalat's lemma.

Given the available sensors and actuators, we have chosen the case of trajectory tracking for motions in depth.

Given equation (3), we get the vector of control input to be:

$$
\boldsymbol{u}=\boldsymbol{T}^{-1} K^{-1} \tau
$$

The (only) parameter to be estimated is $(W-B)$, which represents the difference between the weight $W$ and the buoyancy $B$. The control input of equation (11) will be limited to the degree of freedom around the depth and will be of dimension 1. It is important to emphasize that a good parameter convergence is more guaranteed to occur when the followed trajectory is rich enough to excite the parameters under study (Slotine and Weiping [1991]). However, parameters will converge to a set of values that allow trajectory following, but these values are not necessarily the "true" ones. Moreover, the parameter vector will be bounded but not necessarily convergent. The gains of this controller have been tuned similarly to the case of the PD controller in order to minimize the ISTSE criterion.

\section{REAL-TIME EXPERIMENTAL RESULTS}

In this section the obtained experimental results will be presented and discussed. They result from the application of the 
proposed controllers detailed in section 3 , to the underwater vehicle testbed described in section 2 . The different parameters of the proposed controllers are summarized in table 1. Three experimental scenarios are performed, namely:

Scenario 1: Control of Triton-PR in nominal conditions,

Scenario 2: Robustness towards modeling uncertainties,

Scenario 3: External disturbances rejection.

Table 1. Parameters used in the experiments

\begin{tabular}{cc}
\hline Parameter & Value \\
\hline$k_{z_{1}}$ & 2 \\
$k_{z_{2}}$ & 0.2 \\
$\Gamma$ & 1 \\
$K_{p}$ & 0.8 \\
$K_{d}$ & 0.2 \\
$c_{o}$ & 0.2 \\
$c_{1}$ & 0.1 \\
\hline
\end{tabular}

\subsection{Control of Triton-PR in nominal conditions}

For this scenario, the vehicle is considered without any external disturbances. The obtained experimental results are depicted in Fig.4 and 5 for both controllers, showing their tracking performance.

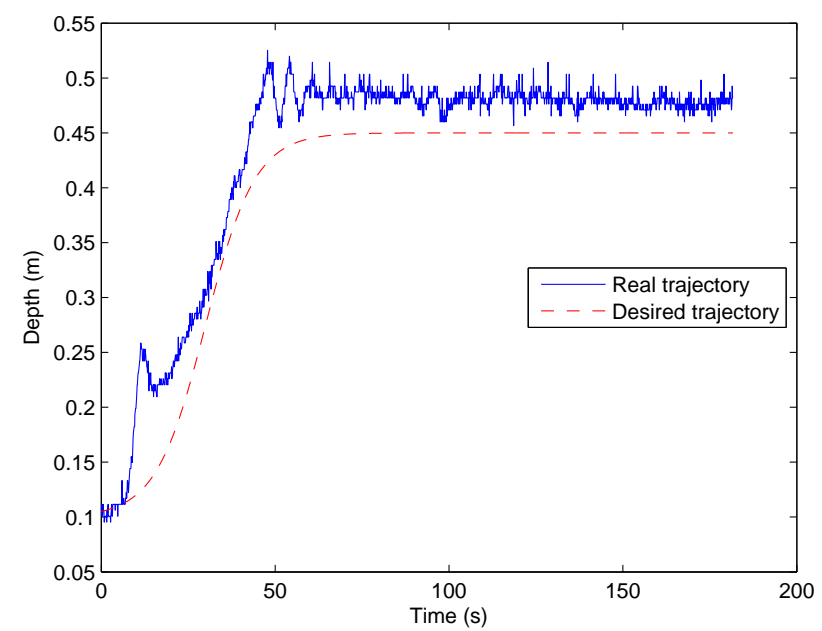

Fig. 4. Experiment of a PD controller on Triton-PR: Time history of the measured z-axis (depth) position (solid line) as well as its corresponding reference trajectory (dashed line)

Figure 5 shows the rapid convergence of the system under the adaptive controller to the trajectory after a suitable gain adjustment while a clear overshoot and more relevant oscillations can be seen in the case of the PD controller. We can also see that the final immersion value obtained with the PD controller is affected by a permanent static error $(3 \mathrm{~cm})$.

\subsection{Robustness towards parameters'change}

The objective of this scenario is to test the robustness of both controllers towards parameters changes of the system. For that purpose one proposes to change the buoyancy by means of

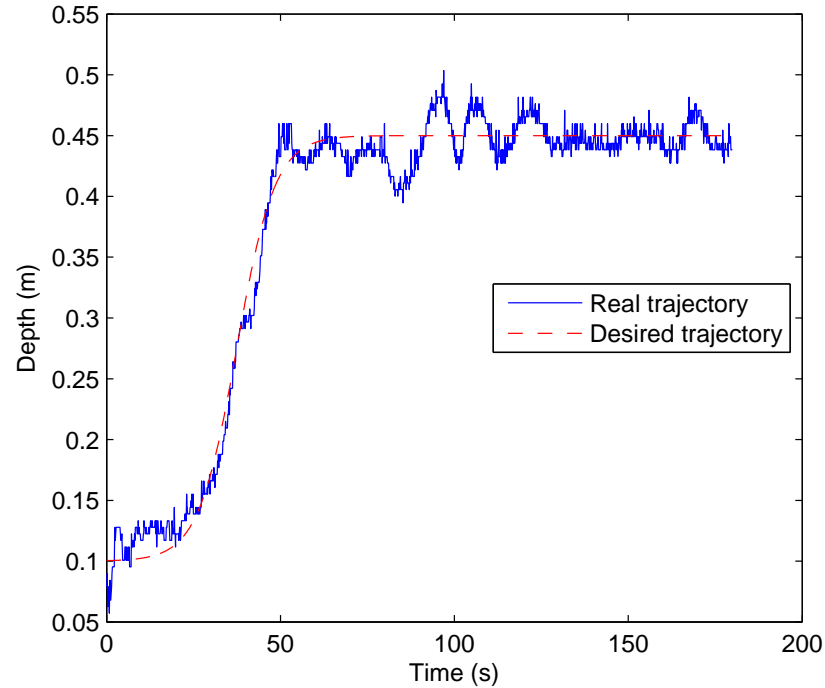

Fig. 5. Experiment of adaptive nonlinear state feedback controller on Triton-PR: Time history of the measured z-axis (depth) position (solid line) as well as its corresponding reference trajectory (dashed line).

adding two small spherical balls of $5.1 \mathrm{~cm}$ diameter, fixed on the top of the vehicle as illustrated in Fig.6. The added floatability to the system can then be estimated to be around $1.4 \mathrm{~N}$.

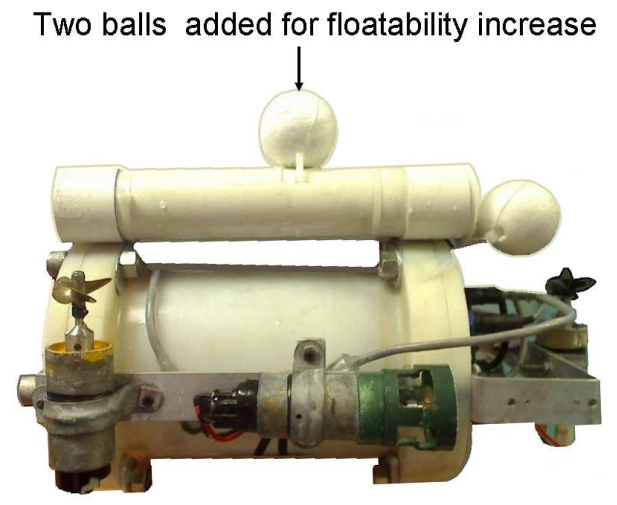

Fig. 6. Two floating balls have been added to the vehicle in order to change its buoyancy.

Once the buoyancy has been changed, the two controllers applied in the first scenario were again tested on the TritonPR. We can clearly note from Fig.7 that the PD was not able to follow the trajectory given that the system changed due to the added buoyancy. The previous gains adjusted for the nominal case tested before were too small to allow the vehicle to reach the desired reference trajectory. By contrast with the PD, the adaptive control law was able to compensate this parameter change. Adaptation of the parameters lasted 120 seconds and then allowed a better trajectory following.

Fig.8 shows the estimation of the parameter $(W-B)$ by the adaptive controller for the nominal case and the case with additional buoyancy. One can note that this parameter varied and 


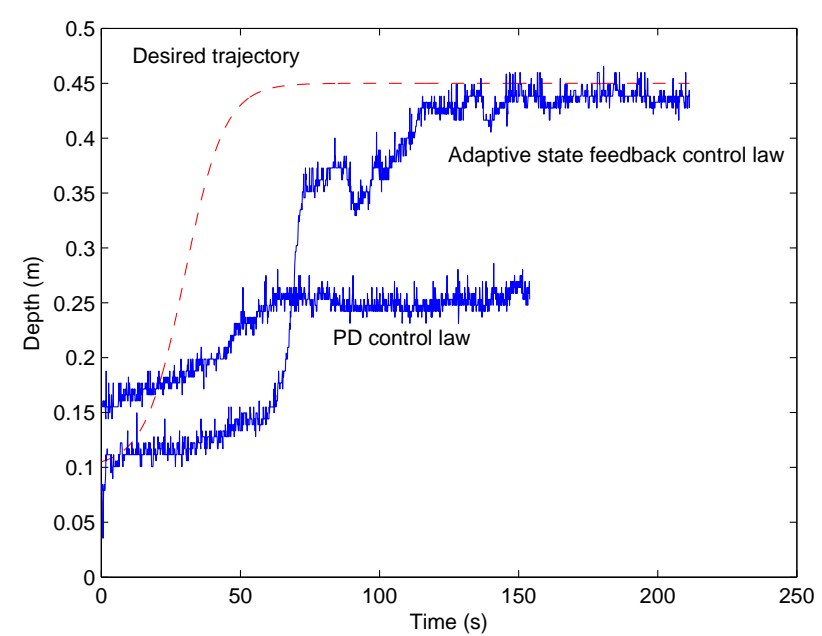

Fig. 7. Time history of the measured $\mathrm{z}$-axis (depth) position obtained with the two studied control laws as well as the reference trajectory used to test the robustness towards a buoyancy change.

adapted to the buoyancy change by converging to a different value. The delay noted in the convergence of the trajectory ( $\simeq 120$ seconds) in Fig.7 coincides with the time needed for the parameter to converge to its steady state estimated value. This convergence time could be shortened by increasing the adaptive gain (however, more attention should be paid since this can lead to instability). Once the parameters have adapted, the vehicle's behavior will be the same as in the nominal case. The difference between the estimated values in the nominal case $(-0.8 \mathrm{~N})$ and the modified case $(-3.5 \mathrm{~N})$ does not correspond to the value $(+1.4 \mathrm{~N})$ of the added buoyancy. This is natural as adaptive controllers do not necessarily need the true values of the estimated parameters to ensure good trajectory following Slotine and Weiping [1991].

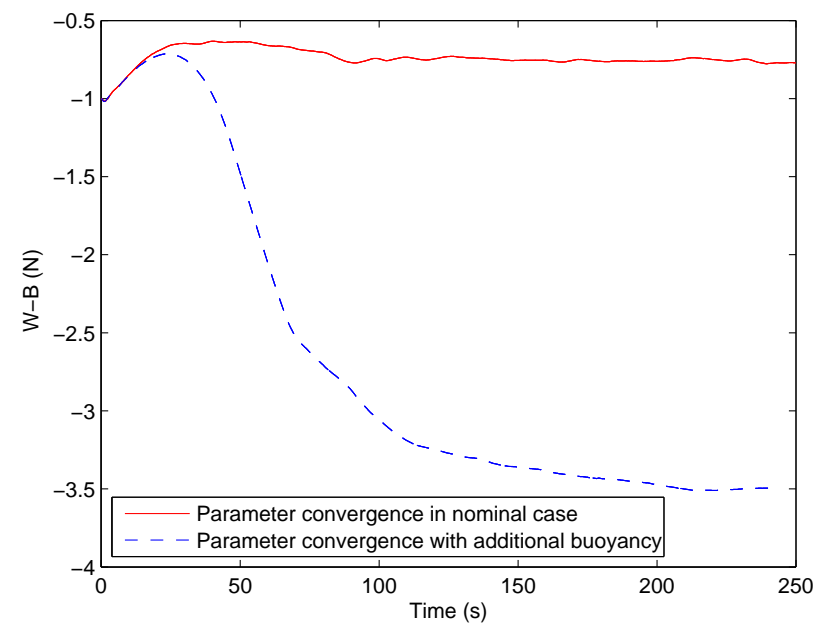

Fig. 8. Time history of the estimated parameter $(W-B)$ resulting from the applied adaptive controller for both scenarios: nominal case and buoyancy change case.

\subsection{External disturbances rejection}

The objective of this scenario is to test the performance of both controllers in presence of external disturbances. The idea is that after the vehicle reached its steady state, a punctual downward force is applied to it.

Fig.9 and 10 below show the recovery of the system after the application of this external disturbance in each of the above two studied cases (PD and Adaptive state feedback controllers). We notice that the PD controller is more sensitive to external disturbances and needs more time to recover than the adaptive controller. Furthermore, it was noticed that with the PD controller an important steady state error was observed.

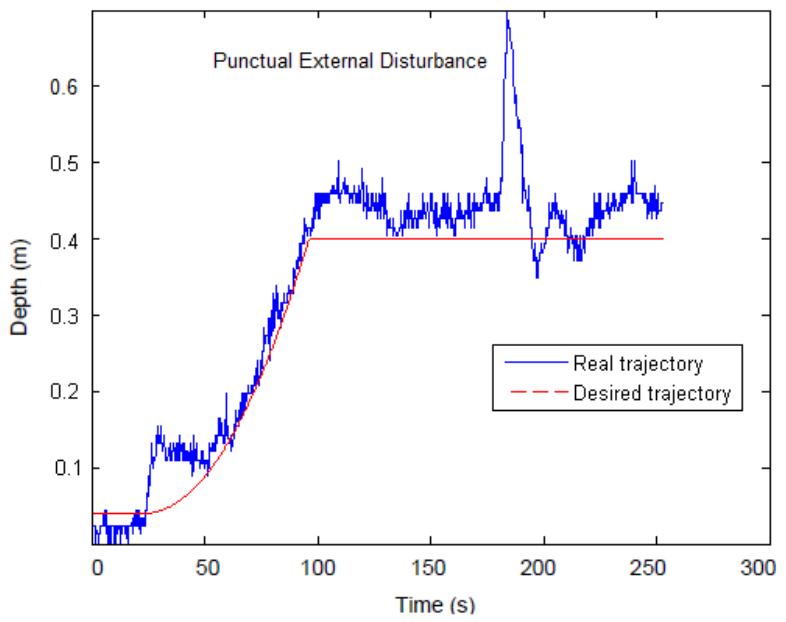

Fig. 9. Time history of the measured z-axis (depth) position (solid line) obtained with the PD controller as well as the reference trajectory (dashed line) used to test its robustness towards a punctual disturbance.

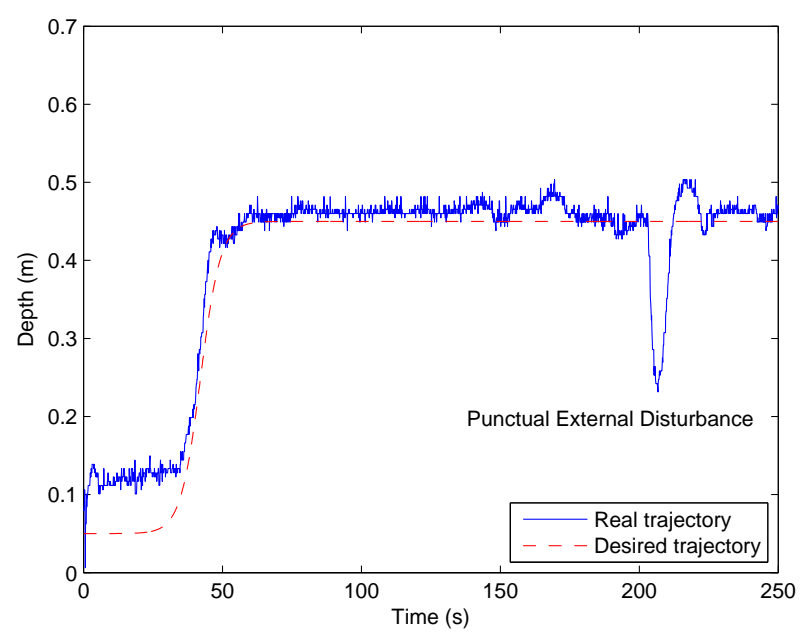

Fig. 10. Time history of the measured z-axis (depth) position (solid line) obtained with the adaptive controller as well as the reference trajectory used (dashed line) to test its robustness towards a punctual disturbance at $t=170 \mathrm{sec}-$ onds.

Remark: The present paper is accompanied with a short video showing the obtained experimental results available at the following link: http://www.youtube.com/watch?v=oqEsXOrpPPk. 


\section{SUMMARY OF COMPARISON BETWEEN THE PROPOSED CONTROLLERS}

From Fig. 4, 5, 7, 9, and 10, we can conclude that the PD controller exhibits important limitations in terms of robustness towards external disturbances and parameter change. Under nominal conditions a better trajectory tracking is observed when the adaptive nonlinear state feedback controller is used. Table 2 below shows the root mean square tracking error at steady state for both scenarios: nominal one as well as the scenario with change in buoyancy.

Table 2. Root Mean Square Error at Steady State (in meters).

\begin{tabular}{lcc}
\hline Controller & Nominal Case & Buoyancy Change \\
\hline PD & 0.032 & 0.20 \\
Adaptive & 0.017 & 0.011 \\
\hline
\end{tabular}

In nominal conditions, the steady state error observed with the adaptive controller was around $4 \%$ compared to $7 \%$ for the PD one. When buoyancy was changed, no important difference was observed on the adaptive controller since the error was around $3 \%$ while $44 \%$ was noticed with the PD controller. Using a PID controller instead of the PD would cancel the steady state error. The gains are not to be adjusted again after the first tuning for both control laws. The performed experiments show clearly that with small model changes the adaptive controller is able to compensate for them and steer the controlled system to the desired trajectory. Some Matlab ${ }^{T M}$ simulations have been performed previously and it was observed that larger changes (e.g $+80 \%$ of buoyancy) would have induced saturation on the thrusters. Hence, the scenarios of the performed experiments were chosen to be in a reasonable range of model changes. Such a small increase in buoyancy can be noted for instance in real environments where salinity is likely to change.

\section{CONCLUSION AND FUTURE WORK}

An experimental comparison study between a PD controller and an adaptive nonlinear state feedback controller has been performed on an underwater vehicle in one degree of freedom. Experimental results have shown that the adaptive controller is largely more robust than the PD controller, since it is able to insure a better trajectory following and has a better compensation for external disturbances. Most importantly, it can adapt online unknown or changing parameters and achieve convergence to the desired trajectory. When parameters are modified, the PD controller behaves poorly. The future work will include more parameters (e.g drag parameters) in the estimation vector of the adaptive controller and the implementation of a PID with a systematic way of tuning the gains for richer trajectories involving more degrees of freedom to induce a more complex nonlinear coupled dynamics.

\section{REFERENCES}

G. Antonelli. An Adaptive Law for Guidance and Control of Remotely Operated Vehicles. In 14th Mediterranean Conference on Control and Automation MED'06, pages 16, Ancona, Italy, June 2006.

G. Antonelli. On the Use of Adaptive/Integral Actions for SixDegrees-of-Freedom Control of Autonomous Underwater
Vehicles. IEEE Journal of Oceanic Engineering, 32:300312, April 2007.

G. Antonelli, S. Chiaverini, N. Sarkar, and M. West. Adaptive control of an autonomous underwater vehicle: experimental results on ODIN. In Proceedings IEEE International Symposium on Computational Intelligence in Robotics and Automation CIRA'99, pages 64-69, Monterey, CA, 1999.

G. Antonelli, F. Cacciavale, S. Chiaverini, and G. Fusco. A Novel Adaptive Control Law for Autonomous Underwater Vehicle. In Proceedings of the IEEE International Conference on Robotics and Automation ICRA'01, pages 447-452, Seoul, Korea, 2001.

G. Campa, M. Innocenti, and F. Nasuti. Robust control of underwater vehicles: sliding mode control vs. mu synthesis. In Proceedings of the IEEE Oceanic Engineering Society OCEANS'98, pages 1640-1644, Nice, France, 1998.

M. Chang, W. Chang, and H.H Liu. Model-based fuzzy modeling and control for autonomous underwater vehicles in the horizontal plane. Journal of Marine Sciences and Technology, 11:155-163, 2003.

T.I. Fossen. Marine Control Systems:Guidance, Navigation and Control of Ships, Rigs and Underwater Vehicles. Marine Cybernetics, As, Trondheim, 2002.

T.I. Fossen and B.A Foss. Sliding control of MIMO nonlinear systems. Modeling, Identification and Control: A Norwegian Research Bulletin, 12(3):129-138, 1991.

T.I. Fossen and S.I Sagatun. Adaptive control of nonlinear underwater robotic systems. In Proceedings of the IEEE International Conference on Robotic and Automation ICRA'91, pages 1687-1695, Sacramento, CA , USA, April 1991.

A.J. Healey, S.M. Rock, S. Cody, D. Miles, and J.P. Brown. Toward an improved understanding of thruster dynamics for underwater vehicles. IEEE Journal of Oceanic Engineering, 20:354-361, 1995.

T.W. Kim and J. Yuh. A novel neuro-fuzzy controller for autonomous underwater vehicles. In Proceedings of the IEEE International Conference on Robotics and Automation ICRA'99, volume 4, pages 2350-2355, 2001.

D. Maalouf, V. Creuze, and A. Chemori. A novel application of multivariable 11 adaptive control: From design to realtime implementation on an underwater vehicle. In IEEE/RSJ IROS'12, Algarve, Portugal, 2012.

I.S. Shaw. Fuzzy control of industrial systems-theory and applications. Kluwer Academic Publisher, 1998.

J.J Slotine and Li Weiping. Applied Nonlinear Control. Prentice-Hall Int, Englewoods Cliffs, New Jersey, 1991.

D.A. Smallwood and L.L. Whitcomb. The effect of model accuracy and thruster saturation on tracking performance of model based controllers for underwater robotic vehicles: experimental results. In Proceedings if the IEEE International Conference on Robotics and Automation ICRA'02, pages 1081-1087, 2002.

A.R Teel. Global stabilization and restricted tracking for multiple integrators with bounded controls. Systems and control letters, 18:165-171, 1992.

O. Yildiz, A.E. Yilma, and B. Gokalp. State-of-the-Art System Solutions for Unmanned Underwater Vehicles. Radioengineering, 18(4):590-600, 2009.

J. Yuh. Design and Control of Autonomous Underwater Robots: A Survey. Autonomous Robots, 24:7-24, 2000. 